\title{
INVENTARIO RÁPIDO DE LOS VERTEBRADOS TERRESTRES DEL PARQUE NACIONAL "DESEMBARCO DEL GRANMA”, NIQUERO, GRANMA, CUBA
}

\section{Rapid inventory of land-living vertebrate from National Park "Desembarco del Granma", Niquero, Granma, Cuba}

\begin{abstract}
Manuel Iturriaga ${ }^{\text {la, }}$, , Hiram González-Alonso ${ }^{1 \mathrm{~b}}$, Ernesto Palacio ${ }^{2}$ y Carlos Hernández ${ }^{1 \mathrm{c}}$
${ }^{1}$ Instituto de Ecología y Sistemática. Carretera Varona \# 11835/Oriente y Lindero, Rpto Parajón, Boyeros, La Habana; la (1) orcid.org/0000-0003-0231-1366, ${ }^{\text {lb }}$ (i) orcid.org/0000-0003-3545-8487, ${ }^{\text {lc }}$ (i) orcid.org/0000-0003-2952-1777. ${ }^{2}$ Parque Nacional "Desembarco del Granma", Empresa Nacional para la Protección de Flora y Fauna, Niquero, Granma; (1) orcid. org/0000-0003-1002-6768. "Para correspondencia: manueliturriaga@ecologia.cu; manueliturriaga83@gmail.com.
\end{abstract}

\section{RESUMEN}

Se aporta la lista de vertebrados terrestres de cuatro localidades del Parque Nacional "Desembarco del Granma", municipio Niquero, provincia Granma. Las aves presentaron la mayor riqueza de especies (54) y los reptiles el mayor grado de endemismo a nivel de especie y/o subespecie (79 \%). En el caso de los anfibios se reporta Eleutherodactylus tonyi, especie endémica y amenazada, y se discute el estado taxonómico de las poblaciones de $E$. cf. varians de Cabo Cruz. En los reptiles se registra por primera vez Hemidactylus angulatus, especie exótica e invasora; y se discute el estado de conservación de las poblaciones Anolis ruibali, un endémico local. Para las aves se recomienda extender los muestreos a otras épocas del año con el objetivo de registrar la entrada de especies migratorias. En los mamíferos, se registra por primera vez Monophyllus redmani, Chilonatalus macer, Mormoops blainvillei y Pteronotus parnelli para esta localidad. La presencia de una cueva caliente en el sendero Morlotte-Fustete aporta un valor adicional desde el punto de vista ecológico y ameritaría replantear planes de conservación y manejo para el área.

Palabras clave: lista de especies, endemismo local, Cabo Cruz, Cuba oriental.

\section{ABSTRACT}

The checklist of land vertebrates of four localities from National Park "Desembarco del Granma", Niquero municipality, Granma province is reported. The birds showed the highest richness of species (54) and the reptiles the highest degree of endemism at species or subespecies level (79 \%). In the case of amphibians, is reported Eleutherodactylus tonyi, endemic and threatened species, and the taxonomic status of the population of $E$. cf. varians from Cabo Cruz is discuted. In the reptiles, Hemidactylus angulatus, exotic and invasive species, is reported for the first time; and the conservation status of the population of Anolis ruibali, a local endemic, is discuted. For birds is recommend to extend the sampling to another seasons in the year, with the aim to register the entrance of migratory species. In the mammals, Monophyllus redmani, Chilonatalus macer, Mormoops blainvillei and Pteronotus parnelli are reported in this locality for the first time. The presence of a hot cave in Morlotte-Fustete path, provides an additional value from ecological viewpoint and it will deserve to redefines conservation plans and managements for the area. 


\section{INTRODUCCIÓN}

Los métodos de inventarios rápidos de biodiversidad fueron desarrollados en la última década del pasado siglo, con el objetivo de obtener información biológica de un área natural (Mittermeier y Forsyth, 1992). Estos tienen como tareas fundamentales: estimar la biodiversidad de un área de interés en particular e identificar sus posibles amenazas, reconocer áreas de interés o con potencial para la conservación, involucrar a la comunidad local en los planes de conservación y manejo, capacitar al personal técnico en métodos de identificación y monitoreo, y desarrollar políticas de manejo y sustentabilidad (Atherton y Jefferies, 2012; Patrick-Apia, 2014). La riqueza de especies, presencia de endémicos locales, especies raras, especies amenazadas y condiciones naturales del hábitat, son algunos de los criterios usados para identificar áreas para la conservación. De modo general los inventarios rápidos de biodiversidad están limitados a listas de especies, aunque algunas veces pueden incluir información sobre la abundancia (Buckland et al., 2001; Herzog et al., 2002; MacLeod et al., 2011).

En la región oriental de Cuba se han llevado a cabo inventarios rápidos de biodiversidad en las zonas montañosas del Macizo Nipe-Sagua-Baracoa (Fa et al., 2002; Fong et al., 2005a), en la Sierra Maestra (Maceira et al., 2005); Siboney-Juticí (Fong et al., 2005b) y Cajobabo (Iturriaga et al., 2018), este último con énfasis en los vertebrados terrestres. Sin embargo, en el área de Cabo Cruz, municipio Niquero, provincia Granma, donde se encuentra ubicado el Parque Nacional Desembarco del Granma, no existen antecedentes de este tipo de estudio; y aunque se tiene conocimiento sobre los recursos naturales que posee, adolece de una evaluación actual (Palacio et al., 2017). Dicha área protegida es uno de los 194 sitios del archipiélago cubano con categoría de Monumento Nacional y, dentro de esta categoría, está entre los 21 con valores naturales destacados para la conservación (CNAP, 2013). Ubicado en la región suroccidental de la provincia de Granma, presenta una superficie total de 32576 ha, de las cuales 26180 corresponden al área terrestre. Reconocido por la UNESCO como Sitio del Patrimonio Mundial Natural de la Humanidad desde 1999 y además reconocido como área protegida de relevancia para la conservación de vertebrados terrestres, en especial aves (IBA) (Reyes y Cala de la Hera, 2010) y reptiles (CNAP, 2013).

\section{OBJETIVO}

- Realizar un inventario rápido de las comunidades de anfibios, reptiles, aves y mamíferos en el parque Nacional Desembarco del Granma, Niquero, Granma.

\section{MATERIALES Y MÉTODOS}

\section{Áreas de estudio}

El territorio se caracteriza por un promedio anual de temperatura de $25-27{ }^{\circ} \mathrm{C}$ y un promedio anual de precipitaciones de $1200 \mathrm{~mm}$. La homogeneidad relativa del tipo de roca (carso) hace que exista poca diferenciación en el tipo de suelo, siendo la rednzina roja el más abundante, mientras que los suelos esqueléticos se ubican sólo en las áreas de los farallones. Los inventarios fueron realizados en localidad 1: Playa Las Coloradas, $\left(77^{\circ} 41^{\prime} 31.139^{\prime \prime} \mathrm{W}, 19^{0} 55^{\prime} 30.176^{\prime \prime} \mathrm{N}\right.$, datum WGS84), localidad 2: Monte Gordo ( $77^{\circ} 42^{\prime}$ 01.048' W, 1953' 53.916" N, datum WGS84), localidad 3: Sendero El Guafe $\left(77^{\circ} 42^{\prime} 56.999^{\prime \prime}\right.$ W, 190 $51^{\prime} 16.171^{\prime \prime}$ N, datum WGS84) y localidad 4: Sendero Morlotte-Fustete ( $77^{\circ} 32^{\prime} 50.258^{\prime \prime}$ W, 19 $9^{\circ} 51^{\prime} 51.347^{\prime \prime} \mathrm{N}$, datum WGS84) entre el 30 de abril y el 5 de mayo de 2019 (Fig. 1). Playa Las Coloradas es un área costera, compuesta por una zona de costa arenosa con matorral xeromorfo costero y franja de uva caleta (Coccoloba uvifera), y otra zona conformada por una franja de mangle con lagunas interiores. 
La zona de costa arenosa muestra cierto grado de antropización debido a las instalaciones de un campismo que está dentro de dicha área. Monte Gordo se caracteriza por la presencia de un bosque semideciduo, donde el estrato herbáceo está generalmente ausente, salvo en zonas antrópicas. Sendero El Guafe se corresponde con una zona de ecotono entre el bosque semideciduo y el matorral xeromorfo costero, siendo característica la existencia de un bosque semideciduo micrófilo con la presencia de varias cactáceas. Existen afloramientos cársicos que poseen algunas cuevas. El sendero Morlotte-Fustete, está asociado a las terrazas marinas bajas y al borde meridional de los niveles de terrazas altos, en superficie de carso desnudo y parcialmente desnudo. En esta localidad se ubica Cueva del Fustete. La vegetación está conformada por estratos arbustivo y arbóreo, emergentes achaparrados con elementos deciduos mayormente esclerófilo, micrófilos y espinosos. La abundancia de suculentas, palmas y epífitas es notable, con presencia de endémicos locales como: Pictetia mucronata (Fabaceae), Coccothrinax saxicola (Arecaceae), Cordia dumosa (Boraginaceae) y Leptocereus sylvestris (Cactaceae), (Palacio et al., 2017).
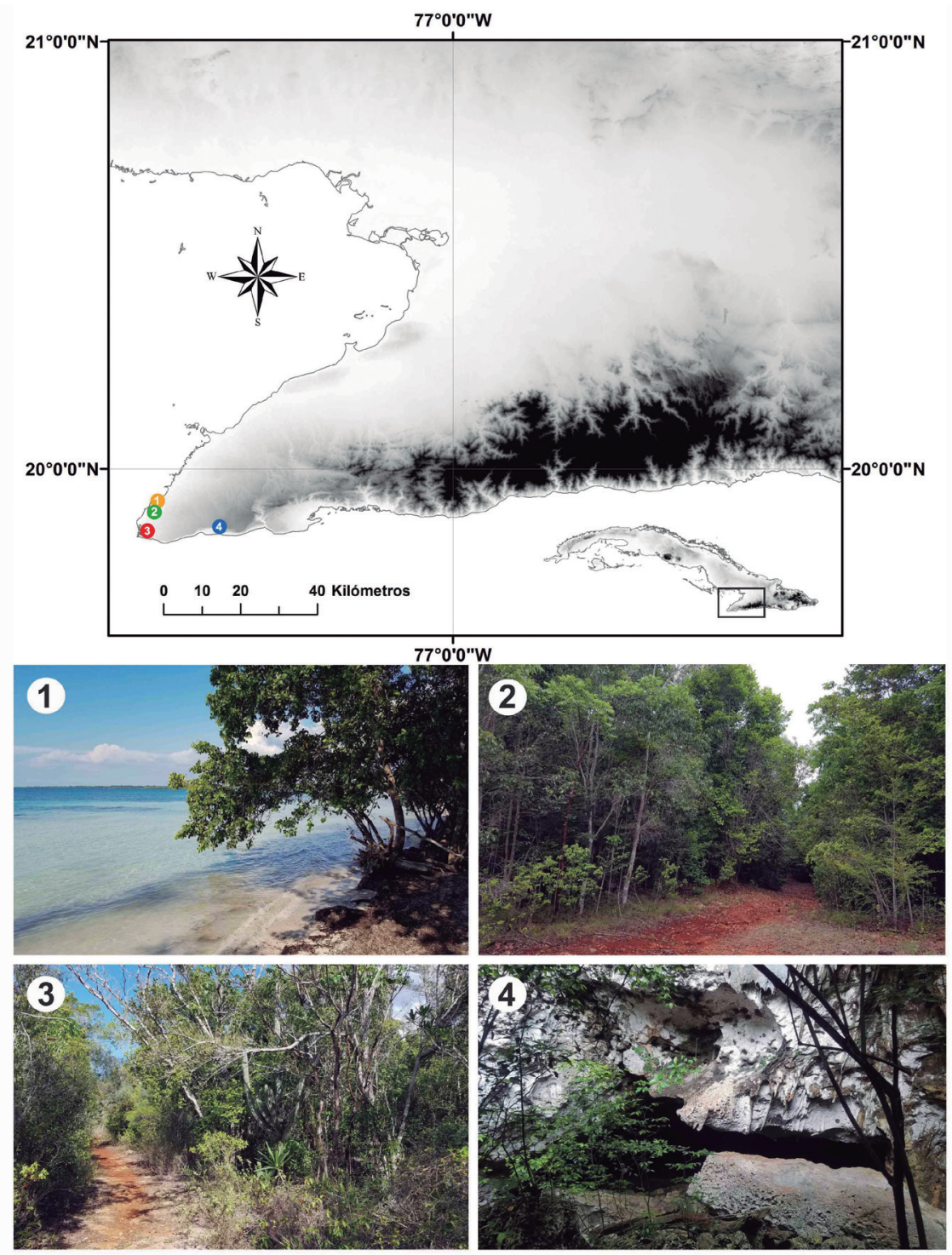

Figura 1. Mapa de las localidades muestreadas en la región de Cabo Cruz, municipio Niquero, provincia de Granma. 1) Playa Las Coloradas, 2) Monte Gordo, 3) sendero El Guafe y 4) sendero Morlotte-Fustete. Fotografías (C M. Iturriaga. 


\section{Muestreos (por grupos zoológicos)}

Se realizó el método de búsqueda libre, excepto para los murciélagos. En Playa Las Coloradas (localidad 1), se siguió por toda la franja de la costa en un transecto lineal de $1500 \mathrm{~m}$ de longitud y $10 \mathrm{~m}$ de ancho, abarcando desde la playa hasta el matorral xeromorfo costero. En la franja de mangle, dentro de la misma localidad, se trabajó en un sendero de hormigón de $1400 \mathrm{~m}$ de longitud y $2 \mathrm{~m}$ de ancho, que atraviesa el manglar y culmina en el mar.

En Monte Gordo, (localidad 2), se muestreó a lo largo de uno de los senderos del área ( $800 \mathrm{~m}$ de longitud y $3 \mathrm{~m}$ de ancho), y abarcando 2 o $3 \mathrm{~m}$ hacia el interior de la vegetación a ambos lados del sendero. En el Sendero El Guafe (localidad 3), se trabajó en el sendero arqueológico que posee $2000 \mathrm{~m}$ de longitud y aproximadamente 3 o $4 \mathrm{~m}$ de ancho. Y en el Sendero Morlotte-Fustete (localidad 4), se muestreó a lo largo del mismo (2500 m de longitud y 2 o $3 \mathrm{~m}$ de ancho), desde el bosque semideciduo hasta las terrazas marinas y farallones. Teniendo en cuenta los diferentes grupos zoológicos, las técnicas de recolecta se muestran a continuación.

Anfibios. Recolecta manual en diferentes microhábitats: hojarasca, bajo piedra, sobre la vegetación y en cuerpos de agua. Para la identificación acústica se dispuso de guías sonoras (Alonso et al., 2007; Díaz y Cádiz, 2008).

Reptiles. Recolecta manual o mediante una vara de 2,4 m de longitud provista de un lazo, en distintos microhábitats: entre hojarasca, bajo piedras y troncos podridos, sobre la vegetación, afloramientos cársicos y farallones. Se utilizaron bolsas de tela y malla para su transportación.

Aves. Fueron identificadas de forma auditiva y visual con el uso de bincoculares $10 \mathrm{X} 42$, auxiliados con guías de campo (Garrido y Kirkconnell, 2002).

Mamíferos. Para los murciélagos se montaron mallas de nieblas (9 m) o se usó una red enmangada cuando se trabajó dentro de las cuevas en el sendero El Guafe (localidad 3) y sendero Morlotte-Fustete (localidad 4). Los registros de roedores se realizaron por identificación visual o la presencia de excrementos en el sendero (Garrido, 2011).

\section{RESULTADOS Y DISCUSIÓN}

El inventario faunístico arrojó un total de 93 especies de vertebrados terrestres en el Parque Nacional Desembarco del Granma: Amphibia (7), Reptilia (19), Aves (54) y Mammalia (13) (Tabla I). En cuanto a la riqueza de especies, los anfibios fueron el grupo con menor valor, mientras que las aves fueron las más numerosas. Sin embargo, los reptiles mostraron el mayor grado de endemismo a nivel de especie y subespecie, $79 \%$ y dentro de este, $37 \%$ corresponde a endémicos locales.

En el caso de los anuros, la mayor diversidad de especies correspondió a Monte Gordo (localidad 2). La misma está conformada por un bosque semideciduo, y presenta altos valores de humedad relativa que propicia un ambiente adecuado para los anfibios. Se registraron especies moradoras del suelo, como Peltophryne peltocephala, Eleutherodactylus atkinsi y E. feichtingeri.

Mientras en la vegetación se pudo escuchar a $E$. cf. varians. Según Díaz y Cádiz (2008), las poblaciones de Cabo Cruz de esta última especie, necesitan una revisión taxonómica, ya que muestran diferencias en cuanto al tamaño corporal y patrones de manchas; y las llamadas de anuncio son muy parecidas a E. staurometopon, taxón perteneciente a la Isla de la Juventud. Sin embargo, estos autores decidieron seguir tratándola como 
E. cf. varians, a diferencia de Alonso et al. (2007) que la definieron como una especie arborícola no descrita (Eleutherodactylus sp.) afín a E. ionthus. Es muy probable que estudios moleculares futuros, y necesarios, arrojen que estas poblaciones corresponden a una nueva especie. En el Caribe, los anfibios muestran altos niveles de endemismo local, y a su vez categorías de amenaza críticas (Hedges y Díaz, 2011). Eleutherodactylus tonyi es una de esas especies endémicas con categoría de amenaza (Rodríguez, 2012). Esta fue observada en este estudio dentro de la Cueva del Fustete (localidad 4). Dicha rana presenta un área de ocupación restringida a $16 \mathrm{~km}^{2}$, lo cual la hace muy vulnerable a los disturbios naturales o antropogénicos (Rodríguez, 2012). Aunque fue reportada inesperadamente para la zona de Gibara, en la provincia de Holguín (Díaz et al., 2014), ampliando su ámbito de distribución a más de $200 \mathrm{Km}$ de distancia, es escaso el conocimiento acerca de ella. Estudios sobre su historia natural son necesarios para futuros programas de monitoreo y mantenimiento en cautiverio (Rodríguez, 2012).

En el caso de los reptiles, la mayor riqueza de lagartos fue registrada en Playa Las Coloradas y El sendero El Guafe. En ambos sitios se observaron especies rastreras del suelo, como Leiocephalus carinatus y Pholidoscelis auberi, y moradoras de troncos, como Anolis angusticeps y $A$. argenteolus.

Playa Las Coloradas (localidad 1) alberga especies asociadas a zonas antropizadas e instalaciones humanas. La presencia de una base de campismo y viviendas cercanas, ofrecen sitios de refugio y percha, tanto para especies diurnas ( $A$. allisoni y A. sagrei), como nocturnas (Hemidactylus angulatus y Sphaerodactylus argus). Estas dos últimas, son especies exóticas que conviven con el hombre dentro de las casas y aprovechan las lámparas de luz artificial para capturar insectos (Henderson y Powell, 2009; Borroto-Páez et al., 2015). En este estudio, esta localidad constituye el registro más suroccidental de $H$. angulatus para la región de Cabo Cruz. El reporte anterior era para el poblado de Niquero, a una distancia de alrededor de $17,3 \mathrm{~km}$ (Díaz, 2014). Hasta el momento no se tiene conocimiento del efecto dañino que puedan acarrear estas dos especies para la fauna nativa del área. Borroto-Páez et al. (2015) determinaron que su impacto en el archipiélago cubano era de consideraciones menores para $H$. angulatus y mínimas para S. argus. No obstante, estos autores plantearon que es muy probable que ambas compitan con especies nativas de menor tamaño asociadas al hombre y sugirieron implementar planes de monitoreo para darles seguimiento.

Por otra parte, el sendero El Guafe (localidad 3), al constituir una zona de ecotono entre el bosque semideciduo y el matorral xeromorfo costero, ofrece una mayor variedad de sitios de percha y refugio para los lagartos, en especial del género Anolis. En esta localidad se registró el mayor número de especies. Algunas de ellas son endémicos locales, como A. equestris verreonensis, A. guafe y A. ruibali. Esta última constituye además una especie rara de la cual apenas se tiene conocimiento (Henderson y Powell, 2009). Navarro (2012) le asignó la categoría de vulnerable, debido a su distribución regional y fragmentada, con un área de ocupación de $8 \mathrm{~km}^{2}$ y tamaño poblacional desconocido.

Entre las amenazas principales se encuentran la pérdida de hábitat por la deforestación y la agricultura, así como la sequía y las penetraciones del mar; y recomienda realizar monitoreos que garanticen datos adicionales de su historia natural para la implementación de planes de conservación y manejo. 
Tabla I. Lista de especies y subespecies de vertebrados terrestres del Parque Nacional Desembarco del Granma

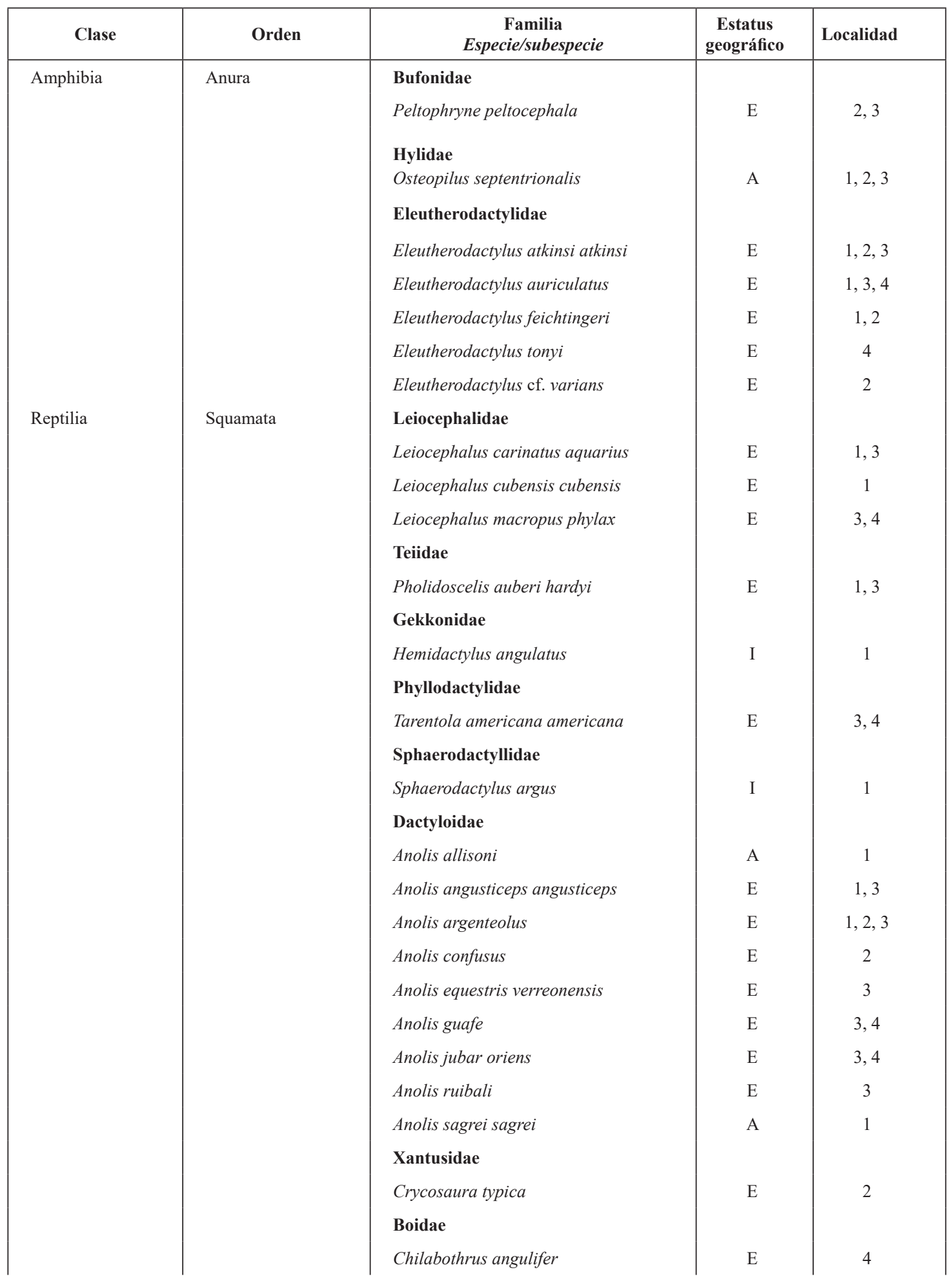




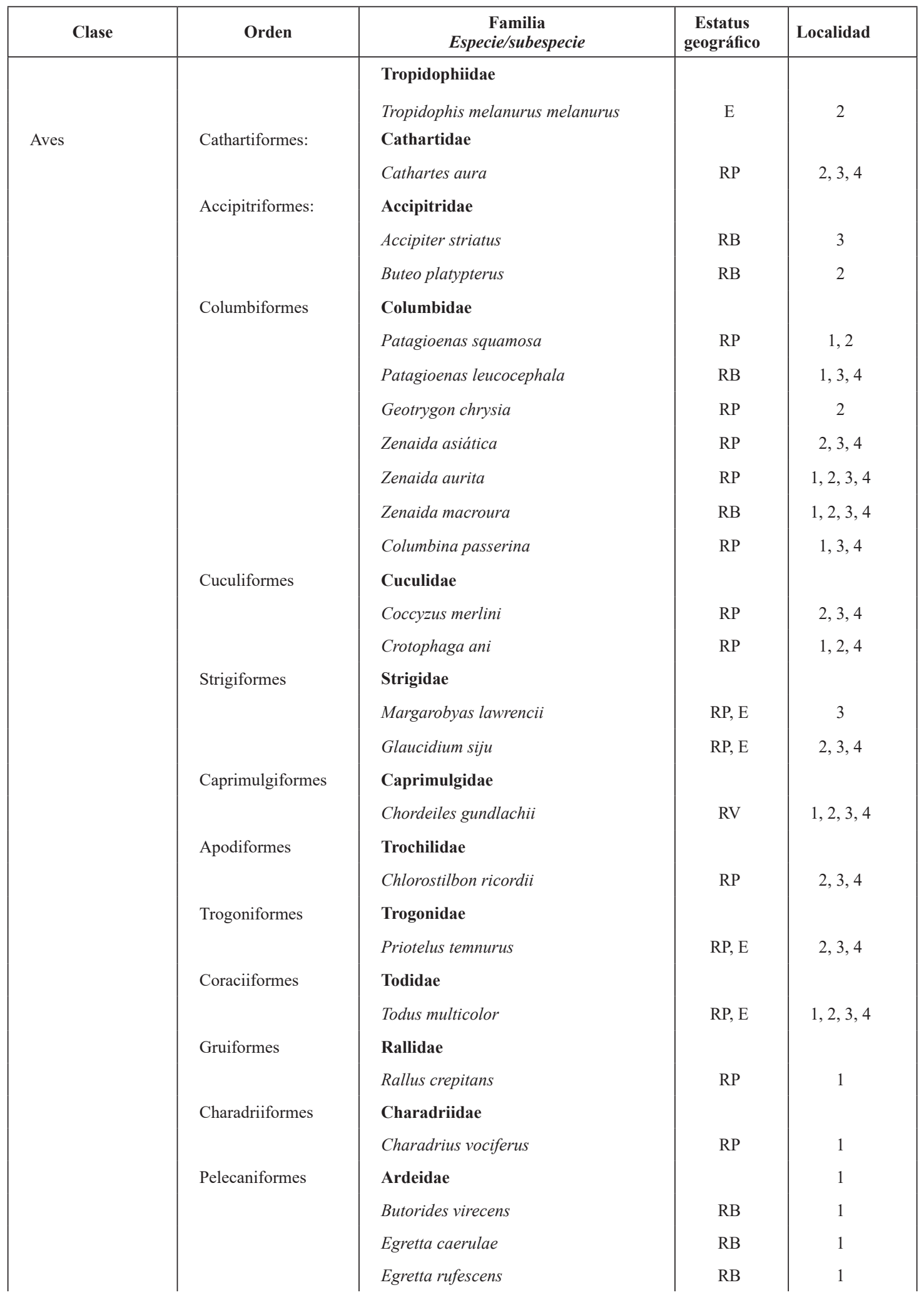




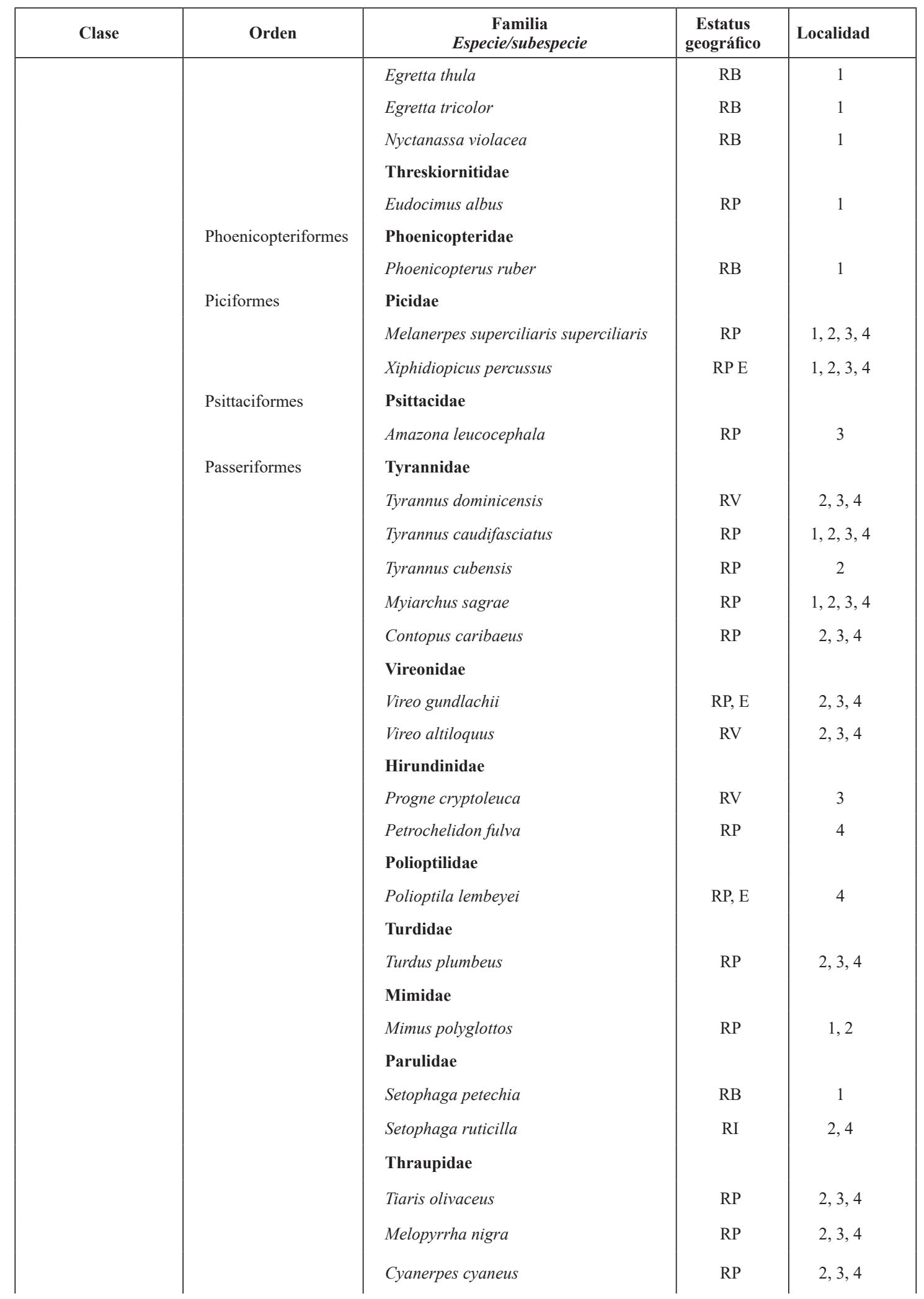




\begin{tabular}{|c|c|c|c|c|}
\hline Clase & Orden & $\begin{array}{c}\text { Familia } \\
\text { Especie/subespecie }\end{array}$ & $\begin{array}{l}\text { Estatus } \\
\text { geográfico }\end{array}$ & Localidad \\
\hline Mammalia & Chiroptera & $\begin{array}{l}\text { Spindalidae } \\
\text { Spindalis zena } \\
\text { Teretistridae } \\
\text { Teretistris fornsi } \\
\text { Icteridae } \\
\text { Quiscalus niger } \\
\text { Ptiloxena atroviolacea } \\
\text { Molothrus bonariensis } \\
\text { Icterus melanopsis } \\
\text { Phyllostomidae } \\
\text { Artibeus jamaicensis parvipes } \\
\text { Brachyphylla nana } \\
\text { Macrotus waterhousei minor } \\
\text { Monophyllus redmani clinedaphus } \\
\text { Phyllonycteris poeyi } \\
\text { Mormoopidae } \\
\text { Mormoops blainvillei } \\
\text { Pteronotus macleayi macleayi } \\
\text { Pteronotus parnelli parnelli } \\
\text { Pteronotus quadridens quadridens } \\
\text { Natalidae } \\
\text { Chilonatalus macer } \\
\text { Nyctiellus lepidus } \\
\text { Capromyidae } \\
\text { Capromys pilorides pilorides } \\
\text { Muridae } \\
\text { Rattus rattus }\end{array}$ & $\begin{array}{c}\text { RP } \\
\text { RP, E } \\
\text { RP } \\
\text { RP, E } \\
\text { RP } \\
\text { RP, E } \\
\text { A } \\
\text { A } \\
\text { A } \\
\text { A } \\
\text { E } \\
\text { A } \\
\text { E } \\
\text { E } \\
\text { A } \\
\text { E }\end{array}$ & $\begin{array}{c}2,3,4 \\
2,3,4 \\
2,3,4 \\
2,3,4 \\
3 \\
1,2,3,4\end{array}$ \\
\hline
\end{tabular}

Estatus geográfico: $\mathrm{E}=$ endémico, $\mathrm{A}=$ autóctona, $\mathrm{I}=$ introducido. Estatus de las aves: $\mathrm{RP}=$ ave residente permanente, $\mathrm{RB}=$ ave residente bimodal, $\mathrm{RI}=$ ave residente invernal, $\mathrm{RV}=$ ave residente de verano. Localidades: $1=$ Las Coloradas, 2 = Monte Gordo, $3=$ Sendero El Guafe, $4=$ Sendero Morlotte-Fustete.

Según Díaz et al. (1998), el área de Cabo Cruz posee la mayor riqueza de especies del género Anolis en el archipiélago cubano; además de ser uno de los sitios con mayor endemismo de reptiles. Navarro y Garrido (2004) consideran a esta zona como un centro importante de diversificación y especiación. Se destacan, además, otros endémicos locales, como $A$. confusus, Cricosaura typica y Leiocephalus macropus phylax (Rodríguez-Schettino et al., 2013).

En las aves, Reyes y Cala de la Hera (2010) reportaron un total de 134 especies, de ellas 78 residentes permanentes, 27 residentes invernales y siete transeúntes. Además, registraron 14 especies endémicas y 10 amenazadas, destacándose el Gavilán Colilargo (Accipiter gundlachi), la Paloma Perdíz (Starnoenas cyanocephala), el Pitirre Real (Tyrannus cubensis) y el Zunzuncito (Mellisuga helenae). Palacio et al. (2017) reportan 129 especies de aves, 
de ellas 62 residentes permanentes, 25 residentes bimodales, ocho residentes de verano, 28 residentes invernales y 23 transeúntes, de las cuales 16 son endémicas y 10 están amenazadas, en las que destacaban la Yaguaza Cubana (Dendrocygna arborea) y el Carpintero Churroso (Colaptes fernandinae). En los muestreos realizados en este trabajo sólo se registraron 54 especies de aves, de ellas 10 endémicas y tres amenazadas, destacándose algunas de las mencionadas anteriormente (Tabla I). Esto se debe al escaso número de muestreos y en el período que se hicieron (abril 30 - mayo 5) donde no se podían detectar las especies migratorias (residentes bimodales y residentes invernales). No obstante, estos resultados confirman que esta área es de gran importancia para la avifauna cubana con más de 130 especies, algunas raras y amenazadas.

En el caso de los mamíferos, se reportaron un total 11 especies de murciélagos para el parque, lo que representa el $42 \%$ de la quiropterofauna cubana (Mancina, 2011). El sendero Morlotte-Fustete (localidad 4) fue la que mostró la mayor riqueza. Dentro esta localidad, específicamente en Cueva del Fustete, se observaron nueve especies de murciélagos pertenecientes a tres familias. De las previamente registradas para el parque en Phyllostomidae y Natalidae, se reportaron por primera vez Monophyllus redmani y Chilonatalus macer, respectivamente (Palacio et al., 2017). Este último, es exclusivo del archipiélago cubano en la actualidad (Tejedor, 2011) y dicha localidad constituye un nuevo reporte de distribución geográfica (Sánchez-Lozada et al., 2018). Por otra parte, en Mormoopidae, fueron registradas por primera vez Mormoops blainvillei y Pteronotus parnelli (Silva, 1979; Sánchez-Lozada et al., 2018).

La presencia de Phyllonycteris poeyi y especies del género Pteronotus sugiere que Cueva del Fustete presenta cámaras calientes, donde se congrega una gran cantidad de individuos, generando así calor metabólico (Silva, 1977; Mancina, 2011). Desde el punto de vista ecológico, la presencia de una cueva caliente en el sendero Morlotte-Fustete es muy productivo para el ecosistema. La congregación de miles de individuos permite tener un tamaño efectivo notable para la prestación de numerosos servicios ecológicos (Rodríguez-Durán, 2009). Los murciélagos son excelentes polinizadores de plantas, dispersores de semillas y controladores de insectos plagas y hematófagos (Mancina, 2011). Esto le brinda un valor adicional al área y es un punto a tener en consideración para futuros planes de acción y manejo al parque. Para las especies que habitan este tipo de cueva, la preservación de colonias viables depende del mantenimiento de un número mínimo de ejemplares $\mathrm{y}$, aunque presentan poblaciones numerosas con altas densidades, son categorizadas como especies vulnerables a extinciones locales (Mancina et al., 2007; Mancina, 2010). Durante el tiempo de estudio la colonia se encontraba en período de gestación, por lo que el monitoreo fue rápido y con la menor cantidad de personal técnico para evitar perturbaciones severas. Los murciélagos cavernícolas en Cuba presentan numerosas amenazas como: alteración de las cuevas para su uso como almacenes y refugios, extracción de guano, en especial las cuevas de calor, y turismo espeleológico desmedido por la falta de regulaciones (Mancina, 2011). Cabe señalar que para la localidad de Monte Gordo fue reportado por primea vez Macrotus waterhousei y M. redmani (Palacio et al., 2017; Sánchez-Lozada et al., 2018).

En el caso de los roedores, la jutía conga (Capromys pilorides) fue avistada en el sendero El Guafe y en el sendero Morlotte-Fustete. Ambas son localidades con un hábitat predominantemente cársico, con presencia de oquedades y pequeñas cuevas. Dentro de los caprómidos cubanos, esta es la especie más terrestre y muestra una marcada preferencia por este tipo de ecosistema (Silva et al., 2007). A pesar de ser la especie con mayor plasticidad ecológica (Borroto-Páez, 2011a) y ninguna categoría de amenaza (Mancina, 2012); la fragmentación y el deterioro del hábitat, la caza furtiva y la presencia de especies invasoras, como perros y gatos jíbaros, están entre las principales amenazas que afectan sus poblaciones (Borroto-Páez y Mancina, 2017). Por otra parte, la rata negra (Rattus rattus) fue avistada en Playa Las Coloradas y Monte Gordo, la primera localidad con un grado notable de antropización por la presencia de instalaciones del campismo y viviendas, mientras que la segunda está mucho más conservada, aunque existen algunas casas aisladas. 
La rata negra está bien distribuida en todo el territorio nacional y es la más asociada a los humanos. Con notables habilidades para trepar, provoca graves daños al ecosistema y la agricultura (Borroto-Páez, 2011b; Borroto-Páez y Mancina, 2017). Por lo tanto, la presencia de esta especie invasora constituye un problema para la estabilidad de la biodiversidad del área y se sugiere tenerla en cuenta en las tareas de manejo del parque, con la búsqueda de medidas que permitan controlar su presencia.

La realización de este inventario rápido permite brindar una aproximación de los valores faunísticos presentes en el área, aun cuando no garantiza una caracterización y estimación completa. La implementación de estudios de monitoreos sistemáticos, con el uso de protocolos de campo, permitirá evaluar a escala temporal las fluctuaciones de las poblaciones presentes en el área (Mancina y Cruz, 2017). Esta tarea debe convertirse en una necesidad de primer orden a nivel de país, más aun en los tiempos actuales, donde el cambio climático y la actividad industrial desmedida del hombre están afectando el estado de salud de los seres vivos a nivel mundial.

\section{AGRADECIMIENTOS}

Agradecemos a Héctor M. Díaz Perdomo del Instituto de Ecología y Sistemática, La Habana y al personal técnico del Parque Nacional, Desembarco del Granma, por la ayuda ofrecida durante el trabajo de campo. A Luis F. de Armas (San Antonio de los Baños, Artemisa), Nayla García y Annabelle Vidal (Instituto de Ecología y Sistemática), por sus revisiones críticas del manuscrito. Así como al editor Carlos Suriel y revisores anónimos que contribuyeron al mejoramiento del mismo. Este estudio fue financiado por el proyecto "Colecciones Zoológicas, su conservación y manejo III”, Ministerio de Ciencia, Tecnología y Medio Ambiente, Cuba.

\section{LITERATURA CITADA}

Alonso, R., A. Rodríguez y M. C. Mena. 2007. Guía sonora de los anfibios de Cuba. Alosa, sons de la natura. Barcelona, España.

Atherton, J. y B. Jefferies. 2012. Guidelines for Undertaking Rapid Biodiversity Assessments in Terrestrial and Marine Environments in the Pacific. SPREP. Apia, Samoa.

Borroto-Páez, R. 2011a. La jutía conga. En: Borroto-Páez, R. y C. A. Mancina (Eds.). Mamíferos de Cuba. UPC Print, Vaasa, Finlandia, 72-81 pp.

Borroto-Páez, R. 2011b. Los mamíferos invasores o introducidos. En: Borroto-Páez, R. y C. A Mancina (Eds.). Mamíferos de Cuba. UPC Print, Vaasa, Finlandia, 220-241 pp.

Borroto-Páez, R. y C. A. Mancina. 2017. Biodiversity and conservation of Cuban mammals: past, present, and invasive species. Journal of Mammalogy, 98 (4): 964-985.

Borroto-Páez, R., R. Alonso, B. A. Fabres y O. Alvarez. 2015. Introduced amphibians and reptiles in the Cuban archipelago. Herpetological Conservation and Biology, 10: 985-1012.

Buckland S. T., D. R. Anderson, K. P. Burnham., J. L. Laake., D. L. Borchers y L. Thomas. 2001: Introduction to distance sampling: estimating abundance of biological populations. Oxford University Press. 
Centro Nacional de Áreas Protegidas (CNAP). 2013. Plan del Sistema Nacional de Áreas Protegidas de Cuba Período 2014-2020. Centro Nacional de Áreas Protegidas, Ministerio de Ciencia, Tecnología y Medio Ambiente. La Habana, 365 pp.

Díaz, L. M. 2014. A new locality record for the Common House Gecko Hemidactylus frenatus Schlegel (Squamata: Gekkonidae) in Cuba, with comments on the other colonizing species of the genus in the island. IRCF Reptiles \& Amphibians, 21: 30-35.

Díaz, L. M. y A. Cádiz. 2008. Guía taxonómica de los anfibios de Cuba. Abc Taxa 4, Bruxelas, Bélgica, 294 pp.

Díaz, L. M., A. Cádiz, S. Villar y F. Bermúdez. 2014. Notes on the Ecology and Morphology of the Cuban Khaki Trope, Tropidophis hendersoni Hedges and Garrido (Squamata: Tropidophiidae), with a New Locality Record. IRCF Reptiles \& Amphibians, 21: 116-119.

Díaz, L. M., N. Navarro y O. H. Garrido. 1998. Nueva especie de Chamaeleolis (Sauria: Iguanidae) de la Meseta de Cabo Cruz, Granma, Cuba. Avicennia, 8-9: 27-34.

Fa, J. E., J. P. Soy, R. Capote, M. Martínez, I. Fernández, A. Ávila, D. Rodríguez, A. Rodríguez, F. Cejas y G. Brull. 2002. Biodiversity of Sierra del Cristal, Cuba: first insights. Oryx, 36: 389-395.

Fong, A., D. Maceira, W. S. Alverson y T. Wachter. 2005a. Cuba: Parque Nacional "Alejandro de Humboldt". Rapid Biological Inventories Report 14. The Field Museum, Chicago: $1-119$.

Fong, A., D. Maceira, W. S. Alverson y J. M. Shopland. 2005b. Cuba: Siboney-Juticí. Rapid Biological Inventories Report 10. The Field Museum, Chicago: 1-210.

Garrido, O. H. 2011. Notas inéditas sobre jutías. En: Borroto-Páez, R. y C. A. Mancina (Eds). Mamíferos en Cuba, UPC Print, Vaasa, Finlandia, 116-121 pp.

Garrido, O. H. y A. Kirkconnell. 2002. Aves de Cuba. Cornell University Press, Ithaca, USA, $176 \mathrm{pp}$.

Hedges, S. B. y L. M. Díaz. 2011. The conservation status of amphibians in the West Indies. 31-47 pp. En: Conservation of Caribbean Island Herpetofaunas Volume 1: Conservation Biology and the Wider Caribbean. Hailey, A., B. Wilson B. y J. Horrocks (Eds). Brill Leiden, The Netherlands.

Henderson, R. W. y R. Powell. 2009. Natural History of West Indian Reptiles and Amphibians. University of Florida Press, Gainesville, 495 pp.

Herzog S. K., M. Kessler y T. M. Cahill. 2002. Estimating species richness of tropical bird communities from rapid assessment data. The Auk, 119: 749-769.

Iturriaga, M., H. M. Díaz, C. Hernández y A. Vidal. 2018. Inventario rápido de los vertebrados terrestres de Cajobabo, Imías, provincia de Guantánamo. Poeyana, 506: 42-47.

Maceira, D., A. Fong, W. S. Alverson y T. Wachter. 2005. Cuba: Parque Nacional La Bayamesa. Rapid Biological Inventories Report 13. The Field Museum, Chicago: 1-81. 
MacLeod R, S. K. Herzog, A. Maccormick, S. R. Ewing, R. Bryce y K. L. Evans. 2011. Rapid monitoring of species abundance for biodiversity conservation: Consistency and reliability of the MacKinnon lists technique. Biological Conservation, 144: 1374-1381.

Mancina, C. A. 2010. Phyllonycteris poeyi (Chiroptera: Phyllostomidae). Mammalian Species, 42 (852): 41-48.

Mancina, C. A. 2011. Introducción a los murciélagos. En: Borroto-Páez, R. y C. A. Mancina (Eds.). Mamíferos de Cuba. UPC Print, Vaasa, Finlandia, 123-133 pp.

Mancina, C. A. 2012. Mamíferos. Introducción. En: González-Alonso, H., L. RodríguezSchettino, A. Rodríguez, C. A. Mancina e I. Ramos-García (Eds). Libro Rojo de los Vertebrados de Cuba. Editorial Academia, La Habana, 269-291 pp.

Mancina, C. A. y D. D. Cruz (Eds). 2017. Diversidad biológica de Cuba: métodos de inventario, monitoreo y colecciones biológicas. Editorial AMA, La Habana, Cuba. 502 pp.

Mancina, C. A., L. Echenique, A. Tejedor, L. García, A. Daniel y M. Ortega. 2007. Endemics under threat: An assessment of the conservation status of Cuban bats. Hystrix, Italian Journal of Mammalogy, 18: 3-15.

Mittermeier, R. A. y A. Forsyth. 1992. Conservation Priorities: The Role of Rap. En: Parker, T. A. y J. L. Carr (Eds.), Rapid Assessment Program: status of forests remnants in the Cordillera de la Costa and Adjacent Areas of South-western Ecuador. Conservation International, Washington.

Navarro, N. 2012. Anolis ruibali. Pp. 132-133. En: González-Alonso, H. L. Rodríguez-Schettino, A. Rodríguez, C. A. Mancina y I. Ramos-García (Eds), Libro Rojo de los Vertebrados de Cuba. Editorial Academia, La Habana, 303 pp.

Navarro, N. y O. H. Garrido. 2004. Especie nueva de Anolis (Sauria: Lacertilia: Iguanidae) de la región Suroriental de Cuba. Solenodon, 4: 85-90.

Palacio, E., S. Calaña, R. Esvalona, Y. Cala y C. C. Heredia. 2017. Plan de Manejo del Parque Nacional Desembarco del Granma. Ministerio de la Agricultura. Empresa Nacional para la Protección de la Flora y la Fauna.

Patrick-Apia, B. 2014. Guidelines for undertaking rapid biodiversity assessments in terrestrial and marine environments in the Pacific. SPREP, Wildlands.

Reyes, E. y Y. Cala de la Hera. 2010. Desembarco del Granma CU019. En: Áreas Importantes para la Conservación de las Aves en Cuba. Editora: Susana Aguilar. Editorial Academia: 86-88.

Rodríguez, A. 2012. Anfibios. En: González-Alonso, H., L. Rodríguez-Schettino, A. Rodríguez, C. A. Mancina e I. Ramos-García (Eds). Libro Rojo de los Vertebrados de Cuba. Editorial Academia, La Habana, 55-92 pp.

Rodríguez-Durán, A. 2009. Bat assemblages in the West Indies: the role of caves. En: Fleming, T. H. y P. A. Racey (Eds.). Island bats. University of Chicago Press, Chicago, 265-280 pp.

Rodríguez-Schettino, L., C. A. Mancina y V. G. Rivalta. 2013. Reptiles of Cuba: Checklist and Geographic Distributions. Smithsonian Herpetological Information Service, 144: 1-92. 
Sánchez-Lozada, M., H. V. Rodríguez, H. M. Díaz, J. Monzón, J. M. de la Cruz, A. Hérnández, A. Longueira, A. Espinosa, T. M. Rodríguez-Cabrera, A. Vidal y C. A. Mancina. 2018. Datos de distribución de murciélagos en Cuba: un acercamiento a través de inventarios biológicos rápidos. Poeyana, 507: 76-81.

Silva, G. T. 1977. Algunos aspectos de la selección de hábitat en el murciélago Phyllonycteris poeyi Gundlach in Peters 1861 (Mammalia: Chiroptera). Poeyana, 168: 1-10.

Silva, G. T. 1979. Los murciélagos de Cuba. Editorial Academia. La Habana. 423 pp.

Silva, G. T., W. Suárez y S. Díaz. 2007. Compendio de los mamíferos terrestres autóctonos de Cuba vivientes y extinguidos. Ediciones Boloña. 465 pp.

Tejedor, A. 2011. Systematics of Funnel-eared bats (Chiroptera: Natalidae). Bulletin of the American Museum of Natural History, 353: 1-140.

[Recibido: 23 de octubre, 2019. Aceptado para publicación: 21 de abril, 2020] 